\title{
Truncation error calculation based on Richardson extrapolation for variable-step collaborative simulation
}

\author{
ZHANG HeMing ${ }^{*}$, LIANG SiLü ${ }^{2}$, SONG ShiJi ${ }^{*}$ \& WANG HongWei ${ }^{3}$ \\ ${ }^{1}$ Department of Automation, TNList, Tsinghua University, Beijing 100084, China; \\ ${ }^{2}$ Computer and Information Management Center, Tsinghua University Beijing 100084, China; \\ ${ }^{3}$ Engineering Design Centre, Engineering Department, Cambridge University, Cambridge CB2 1PZ, UK
}

Received September 28, 2010; accepted January 11, 2011

\begin{abstract}
Collaborative simulation is an effective approach to performing simulation analysis for complex systems by integrating models developed for different engineering disciplines. Collaborative simulation issues include the modeling of coupled multidisciplinary systems, and the simulation running time integration of these models that are solved parallelly. Estimation of the local truncation error of coupling models is the key to solve multidisciplinary collaborative simulation problem, which is actually used to restrict the simulation step. This paper presents a variable-step method based on Richardson extrapolation for calculating the local truncation error to solve collaborative simulation problem of multidisciplinary coupling models. Formulas for estimating the local truncation error are derived through theoretical analysis by using integration methods and interpolation technologies, respectively. The simulation experiments are illustrated to validate the accuracy and efficiency of proposed collaborative simulation algorithm in comparison with the usual combinative algorithm.
\end{abstract}

Keywords multidisciplinary systems, collaborative simulation, variable-step algorithm, error estimation

Citation Zhang H M, Liang S L, Song S J, et al. Truncation error calculation based on Richardson extrapolation for variable-step collaborative simulation. Sci China Inf Sci, 2011, 54: 1238-1250, doi: 10.1007/s11432-011-4274-z

\section{Introduction}

Collaborative simulation is becoming a popular approach to modeling and simulation of complex systems. It aims to achieve optimal solutions of complex systems by taking into account the coupling problems between multidisciplinary subsystems, and has been widely used in engineering domains [1-3]. The challenges for collaborative simulation include the multidisciplinary modeling, collaborative interaction, time-synchronized running and simulation platform, where a variety of disciplinary simulation tools are handled dynamically in a distributed environment [4]. Some researches have been done with focuses on the model-driven modeling approach $[5,6]$, the distributed interaction mechanisms between simulation models [7], and the development of collaborative simulation platform [8, 9]. Essentially, distributed collaborative simulation can be viewed as a process of parallelly solving equations of subsystems and synchronously exchanging run-time data with an appropriate time step. The main concerns include

\footnotetext{
*Corresponding authors (email: hmz@tsinghua.edu.cn, shijis@tsinghua.edu.cn)
} 
stability, accuracy, or the combination of both [10]. Shome et al. [11] proposed a partitioned RungeKutta method by combining a multi-rate integration method with partitioned integration. Arnold [12, 13] studied the numerical methods for simulation and multi-rate integration in applied dynamics. The issues addressed in their works include the selection and control of step size, the processing of exchanged data, and the computation of ill-structured models. In numerical calculation, large-scale ordinary differential equations are often solved by dividing them into several subsystems with moderate scale. The classical algorithms for such division include the multi-rate method by Gear et al. [14] and the combinative algorithm by Fei et al. [15]. These algorithms provide a theoretical basis for the numerical solving of large-scale complex models, and their convergence has been proved [16]. However, the coupling vectors between subsystems in collaborative simulation are usually complex; thus it is urgent to develop efficient and accurate algorithms.

The algorithms currently available for collaborative simulation mainly fall into the category of combinative algorithms with a fixed united step; that is, a fixed global step is used throughout the whole simulation process, whereas each subsystem is simulated by its internal variable-step integration algorithms [17]. Actually, some efficient variable-step algorithms have been developed for, and applied in, the solving of a single system model [18], though similar algorithms have not been developed for collaborative simulation. The core of such algorithms is to determine the variable step size by estimating the calculation errors. Some researchers investigated this field. For example, Schiehlen et al. [19] proposed a variable-step algorithm for collaborative simulation based on Richardson extrapolation and the embedding method, using an iterative way to estimate the errors of each subsystem and then determine the step size. In order to address the issue of calculation divergence caused by the algebraic loops in the coupling models, Kübler et al. [20] developed a method to improve the stability of the algorithm based on united step by using the quasi-Newton iteration method or adding filters in the models to eliminate the algebraic loops. However, such iterative algorithms have high computational complexity, and fail to satisfy the requirements for real-time simulation.

The paper presents our research on developing a variable-step collaborative simulation algorithm which is based on the idea of Richardson extrapolation in the simulation of a single system. Some theoretical foundations are given first. Then the analysis and calculation of truncation error in solving collaborative simulation problems are investigated by extending and utilizing the current methods to multidisciplinary coupling systems, the truncation error formulas of the coupling models are derived, with different conditions taken into account, such as the different orders of integration methods and the interpolation methods. Finally, simulation experiments are demonstrated to present efficiency and accuracy of proposed algorithm comparing with combinative algorithm in [17].

The remaining part of this paper is arranged as follows. Section 2 introduces the basic theory of the algorithm. Section 3 presents a collaborative computing method for coupling systems. Section 4 develops calculation methods for local truncation error of the collaborative simulation system. Section 5 derives the estimation formulas of the local truncation error for a coupling system model. Section 6 implements the simulation experiments for evaluation of the proposed method. Section 7 gives conclusions of the paper.

\section{Basic theory of the algorithm}

\subsection{Richardson extrapolation}

Assume that a system can be described by

$$
\frac{d z(t)}{d t}=f(z(t), t), \quad z\left(t_{0}\right)=z_{0}, \quad z \in \mathbb{R}^{M+N}
$$

where $t$ is time, and $z(t)$ is the state vector in the $(M+N)$-dimensional space.

To perform numerical integration for such a system, Richardson developed simple formula to estimate local truncation errors using extrapolation. The calculation procedures are shown in Figure 1 [17]. 


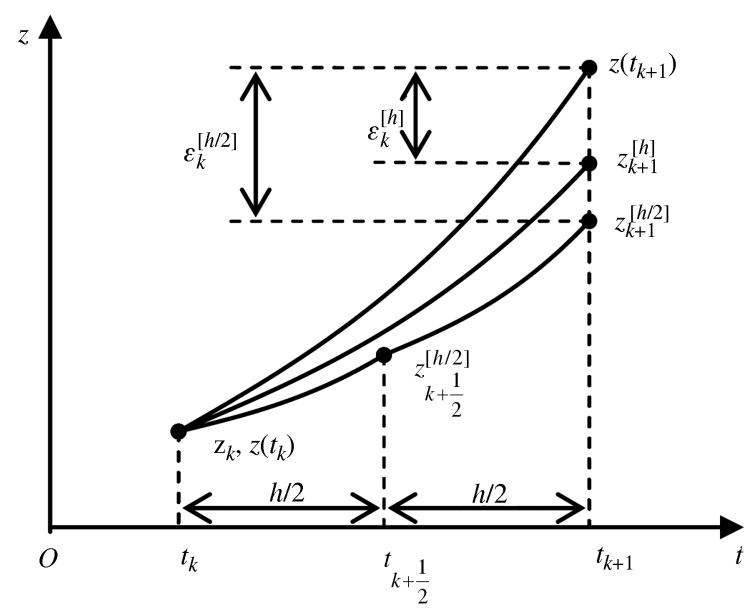

Figure 1 Richardson extrapolation.

$\psi(z, t, h)$ is the increment function of the integration algorithm, and $z_{k}$ is the value of function $z(t)$ calculated at $t_{k}$. The step size is $h$, and the result at $t_{k}+h$ is $z_{k+1}^{[h]}$ which can be given by

$$
z_{k+1}^{[h]}=z_{k}+h \psi\left(z_{k}, t_{k}, h\right) .
$$

Then by using step size $h / 2$, the result of $z_{k+1}^{[h / 2]}$ can be calculated in two steps as follows:

$$
\begin{aligned}
& z_{k+\frac{1}{2}}^{[h / 2]}=z_{k}+\frac{h}{2} \psi\left(z_{k}, t_{k}, \frac{h}{2}\right), \\
& z_{k+1}^{[h / 2]}=z_{k+\frac{1}{2}}^{[h / 2]}+\frac{h}{2} \psi\left(z_{k+\frac{1}{2}}^{[h / 2]}, t_{k}+\frac{h}{2}, \frac{h}{2}\right) .
\end{aligned}
$$

When the order of the integration algorithm is $p$ (this means that the order of the local truncation error is $p+1)$, from $(2)$ and (3), we have

$$
\varepsilon_{k}^{[h]}=z\left(t_{k+1}\right)-z_{k+1}^{[h]}=z\left(t_{k+1}\right)-z_{k}-h \psi\left(z_{k}, t_{k}, h\right)=c\left(t_{k}\right) h^{p+1}+O\left(h^{p+2}\right),
$$

such that $z\left(t_{k+1}\right)=z_{k+1}^{[h]}+c\left(t_{k}\right) h^{p+1}+O\left(h^{p+2}\right)$,

$$
\begin{aligned}
\varepsilon_{k}^{[h / 2]} & =z\left(t_{k+1}\right)-z_{k+1}^{[h / 2]}=z\left(t_{k+1}\right)-z_{k}-\frac{h}{2} \psi\left(z_{k}, t_{k}, \frac{h}{2}\right)-\frac{h}{2} \psi\left(z_{k+\frac{1}{2}}^{[h / 2]} t_{k}+\frac{h}{2}, \frac{h}{2}\right) \\
& =z\left(t_{k+1}\right)-z_{k+\frac{1}{2}}-\frac{h}{2} \psi\left(z_{k+\frac{1}{2}}^{[h / 2]}, t_{k}+\frac{h}{2}, \frac{h}{2}\right)+z_{k+\frac{1}{2}}-z_{k}-\frac{h}{2} \psi\left(z_{k}, t_{k}, \frac{h}{2}\right) \\
& =c\left(t_{k+\frac{1}{2}}\right)\left(\frac{h}{2}\right)^{p+1}+c\left(t_{k}\right)\left(\frac{h}{2}\right)^{p+1}+O\left(h^{p+2}\right) \\
& =c\left(t_{k}\right) \frac{h^{p+1}}{2^{p}}+O\left(h^{p+2}\right) .
\end{aligned}
$$

So, $z\left(t_{k+1}\right)=z_{k+1}^{[h / 2]}+c\left(t_{k}\right) \frac{h^{p+1}}{2^{p}}+O\left(h^{p+2}\right)$.

As $z\left(t_{k+1}\right)=z_{k+1}^{[h]}+c\left(t_{k}\right) h^{p+1}+O\left(h^{p+2}\right)$, there holds $c\left(t_{k}\right) \approx \frac{1}{h^{p+1}\left(1-2^{-p}\right)}\left(z_{k+1}^{[h / 2]}-z_{k+1}^{[h]}\right)$.

The formulas to estimate local truncation error for a single system can then be derived as

$$
\begin{aligned}
& \varepsilon_{k}^{[h]} \approx \frac{1}{1-2^{-p}}\left(z_{k+1}^{[h / 2]}-z_{k+1}^{[h]}\right), \\
& \varepsilon_{k}^{[h / 2]} \approx \frac{1}{2^{p}-1}\left(z_{k+1}^{[h / 2]}-z_{k+1}^{[h]}\right) .
\end{aligned}
$$

Therefore, the local truncation error in each single step can be estimated in the calculation at steps $h$ and $h / 2$. By comparing the error estimated and the control error that is set to ensure the accuracy 
of calculation, the step size can be controlled and the variable-step algorithm can be enabled. However, Richardson extrapolation method cannot be simply used in the collaborative simulation with multiple coupling models.

\subsection{Lagrange interpolation polynomial}

Assume the values of function $f(t)$ at the different nodes $t_{0}, t_{1}, \ldots, t_{n}$ in interval $[a, b]$ are $y_{0}, y_{1}, \ldots, y_{n}$. Then an $n$-order Lagrange interpolation polynomial $L_{n}(t)$ can be formulated as follows.

$$
L_{n}(t)=y_{0} l_{0}(t)+y_{1} l_{1}(t)+\cdots+y_{n} l_{n}(t)=\sum_{i=0}^{n} y_{i} l_{i}(t)
$$

where $l_{i}(t)=\frac{\left(t-t_{0}\right)\left(t-t_{1}\right) \cdots\left(t-t_{i-1}\right)\left(t-t_{i+1}\right) \cdots\left(t-t_{n}\right)}{\left(t_{i}-t_{0}\right)\left(t_{i}-t_{1}\right) \cdots\left(t_{i}-t_{i-1}\right)\left(t_{i}-t_{i+1}\right) \cdots\left(t_{i}-t_{n}\right)} \cdot l_{0}(t), l_{1}(t), \ldots, l_{n}(t)$ are termed the $n$-order interpolation basis function at nodes $t_{0}, t_{1}, \ldots, t_{n}$.

Obviously, $L_{n}(t)$ is a polynomial and its order is no more than $n$, and satisfies $L_{n}\left(t_{i}\right)=y_{i}, i=$ $0,1, \ldots, n$.

\section{Collaborative computing method for coupling systems}

\subsection{Description of a coupling system model}

Generally, a complex engineering system involves a number of disciplinary domains, e.g. kinematics and multi-body dynamics, which can be modeled as a set of continuous differential equations. Moreover, a complex system needs to be divided into several coupling subsystems, and is then analyzed by taking into account the cooperation between them.

Simply, a system with two coupling subsystems is analyzed in this section, and characteristics of more complex system can be identified similarly. For the system model described in (1), the state vector $z(t)$ is divided into two subsystem state vectors $z_{1}(t)$ and $z_{2}(t)$. Likely, the system function $f(z(t), t)$ is also replaced by two functions and each of which represents a subsystem, expressed as

$$
\begin{aligned}
& \left\{\begin{array}{l}
\frac{d z_{1}(t)}{d t}=f_{1}\left(z_{1}(t), Y_{21}(t), t\right), z_{1}\left(t_{0}\right)=z_{10}, z_{1} \in \mathbb{R}^{M}, \\
Y_{12}(t)=y_{1}\left(z_{1}(t), Y_{21}(t), t\right), Y_{12} \in \mathbb{R}^{S}
\end{array}\right. \\
& \left\{\begin{array}{l}
\frac{d z_{2}(t)}{d t}=f_{2}\left(z_{2}(t), Y_{12}(t), t\right), z_{2}\left(t_{0}\right)=z_{20}, z_{2} \in \mathbb{R}^{N} \\
Y_{21}(t)=y_{2}\left(z_{2}(t), Y_{12}(t), t\right), Y_{21} \in \mathbb{R}^{T}
\end{array}\right.
\end{aligned}
$$

where $z_{1}(t)$ is the state vector of $M$-dimensional space of subsystem $\mathrm{S}_{1} ; z_{2}(t)$ is the state vector of $N$ dimensional space of subsystem $\mathrm{S}_{2} ; Y_{12}(t), Y_{21}(t)$ are a pair of coupling vectors, representing the output vectors of subsystem models and the input vectors of subsystem models, respectively.

\subsection{Collaborative computing method for a coupling system model}

For the coupling model in (9) and (10), collaborative computing needs to be utilized, and variable-step integration algorithm can improve the efficiency of collaborative simulation. As shown in Figure $2, H_{i}$ is the step size of two coupling subsystems involved in collaborative simulation, and the value of $H_{i}$ is determined by the local truncation error of the coupling system. For each $H_{i}$, the start and end points of this simulation step are taken as two endpoints of the interval $\left[t_{i}, t_{i+1}\right]$, and data exchange takes place by assigning value of $Y_{12}(t), Y_{21}(t)$ at these points. Denote the time interval of whole collaborative simulation by $\left[t_{0}, t_{N}\right]$. Then time points $t_{i+1}=t_{i}+H_{i}, i=0,1,2, \ldots, N-1$ are the endpoints of the $N$ sub-intervals. Inside each $H_{i}$, integration is performed for subsystems $\mathrm{S}_{1}$ and $\mathrm{S}_{2}$ by using (9) and (10).

For the numerical calculation of (9) and (10), the input of each subsystem model is obtained via interpolation on the output of another subsystem model. The interpolation methods such as Lagrange 


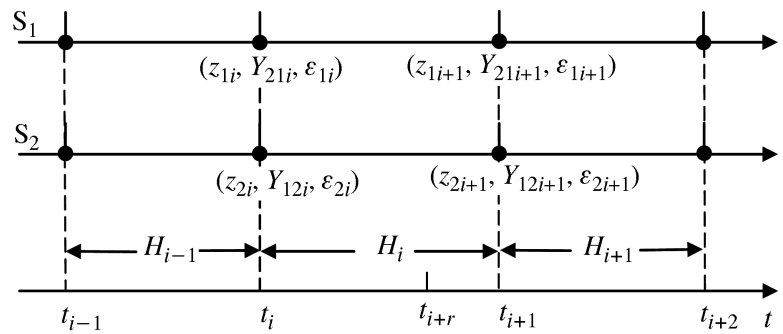

Figure 2 Collaborative computing for a coupling system model.

polynomial or Hermite polynomial can be applied to solve this problem. Assume that the calculation values of $Y_{12}(t), Y_{21}(t)$ at each $H_{i}$ are $Y_{12 k}, Y_{21 k}, k=0,1, \ldots, i$. Let $T_{i}=\left(t_{0}, t_{1}, \ldots, t_{i}\right)^{\mathrm{T}}, Y_{1 i}=$ $\left(Y_{120}^{\mathrm{T}}, Y_{121}^{\mathrm{T}}, \ldots, Y_{12 i}^{\mathrm{T}}\right)^{\mathrm{T}}$ and $Y_{2 i}=\left(Y_{210}^{\mathrm{T}}, Y_{211}^{\mathrm{T}}, \ldots, Y_{21 i}^{\mathrm{T}}\right)^{\mathrm{T}}$. Then the Lagrange interpolation function $I_{Y 1 i}$ $\left(Y_{1 i}, T_{i}\right)(t)$ and $I_{Y 2 i}\left(Y_{2 i}, T_{i}\right)(t)$ can be obtained by pairs $\left(Y_{1 i}, T_{i}\right)$ and $\left(Y_{2 i}, T_{i}\right)$. Mark $\tilde{Y}_{12}(t)=I_{Y 1 i}\left(Y_{1 i}\right.$, $\left.T_{i}\right)(t), \tilde{Y}_{21}(t)=I_{Y 2 i}\left(Y_{2 i}, T_{i}\right)(t)$. Then for the computation going progressively from $t_{i}$ to $t_{i+1}$, we need to determine a proper length of $H_{i}$ i.e. the step size for collaborative simulation based on local truncation error.

\section{Calculation of local truncation error}

\subsection{Method of calculation}

Now, the subsystem $S_{1}$ is used as an example to illustrate the calculation method for local truncation error of a coupling system represented in (9) and (10).

The difference in methods for calculating the local truncation error between subsystem $\mathrm{S}_{1}$ in $(9)$ and a single system in (1) is that input vector $Y_{21 i}$ from subsystem $\mathrm{S}_{2}$ to subsystem $\mathrm{S}_{1}$ at time $t_{i}$ cannot be yielded accurately because there exists the coupling vector $Y_{21}(t)$. As the value $\tilde{Y}_{21}\left(t_{i}\right)$ of interpolation function $\tilde{Y}_{21}(t)$ at time point $t_{i}$ is used to replace $Y_{21 i}$, interpolation error occurs when data exchange takes place between subsystem models.

Denote $\tilde{Y}_{21 i}=\tilde{Y}_{21}\left(t_{i}\right), \tilde{Y}_{21 i+1}=\tilde{Y}_{21}\left(t_{i+1}\right) \cdots$. Other symbols are also denoted in the same way. Assuming $\Delta_{f_{1}}\left(z_{1}\left(t_{i}\right), Y_{21}\left(t_{i}\right), t_{i}, H_{i}\right)$ is the theoretical increment of the integration algorithm used for subsystem $(9)$, we get $z_{1}\left(t_{i+1}\right)=z_{1}\left(t_{i}\right)+H_{i} \Delta_{f_{1}}\left(z_{1}\left(t_{i}\right), Y_{21}\left(t_{i}\right), t_{i}, H_{i}\right)$. Denote by $\psi_{f_{1}}\left(z_{1 i}, \tilde{Y}_{21 i}, t_{i}, H_{i}\right)$ the increment function, i.e. $z_{1 i+1}^{[H]}=z_{1 i}+H_{i} \psi_{f_{1}}\left(z_{1 i}, \tilde{Y}_{21 i}, t_{i}, H_{i}\right)$. Simply, the increment function of one-step integration algorithm is used here. $z_{1 i}$ and $\varepsilon_{1 i}$ are the corresponding calculated value and calculation error of state vector $z_{1}\left(t_{i}\right)$ at time $t_{i} ; z_{1 i+1}$ and $\varepsilon_{1 i+1}$ are the calculated value and calculation error of state vector $z_{1}\left(t_{i+1}\right)$ at time $t_{i+1} ; \varepsilon_{\psi f_{1}}$ is the integration error of subsystem $\mathrm{S}_{1}$ in united step $H_{i}$ from $t_{i}$ to $t_{i+1} ; \varepsilon_{i n t 2}$ is the interpolation error of input vector $\tilde{Y}_{21}$ from subsystem $\mathrm{S}_{2}$ to $\mathrm{S}_{1}$. In order to derive the local truncation error from $t_{i}$ to $t_{i+1}$, cumulated errors are not considered here for convenience. So, we get $z_{1 i}=z_{1}\left(t_{i}\right), Y_{21 i}=Y_{21}\left(t_{i}\right), \tilde{Y}_{21 i}=Y_{21}\left(t_{i}\right)$.

There are a number of different situations for the local truncation error calculation.

(I) The simplest case: The integral method is the Euler method, i.e. 0-order one step method. The input vector of increment function includes only $\tilde{Y}_{21 i}$. Since $\tilde{Y}_{21 i}=Y_{21}\left(t_{i}\right)$, the interpolation error is 0 and the Richardson extrapolation method can be used directly. This simple case is not considered in this paper.

(II) A more complex case: The integral method is the improved Euler method, i.e. 1-order one step method or a multi-step method with any order. The input vector of increment function includes $\tilde{Y}_{21 i+1}$ and $\tilde{Y}_{21 i}$ at the points $i+1, i$, and even $\tilde{Y}_{21 i-1}, \tilde{Y}_{21 i-2}, \ldots, \tilde{Y}_{21 i-n}$ at points $i-1, i-2, \ldots, i-n$ is used in the multi-step method. So, the interpolation error is mainly caused by $\tilde{Y}_{21 i+1}$, which is an extrapolated polynomial using the interpolation data before time $t_{i}$.

(III) A much more general case: The integral method is one step method of higher order like RungeKutta. The input vector of increment function includes not only $\tilde{Y}_{21 i+1}$ and $\tilde{Y}_{21 i}$ at points $i+1, i$, but 
also $\tilde{Y}_{21 i+1 / 2}, \tilde{Y}_{21 i+1 / 3}, \ldots$ at points $i+1 / 2, i+1 / 3, \ldots$, and so on. So the interpolation error comes from multiple sources. This case is also not considered in this paper.

This paper will only consider the calculation of local truncation error in case (II), i.e. improved Euler method. Without loss of generality, the increment of error for input vectors $\tilde{Y}_{21 i+1}, \tilde{Y}_{21 i}, \tilde{Y}_{21 i-1}, \ldots, \tilde{Y}_{21 i-n}$ at the input points $i+1, i, i-1, \ldots, i-n$ can be calculated as follows:

$$
\begin{aligned}
\varepsilon_{1 i+1}^{[H]}= & z_{1}\left(t_{i+1}\right)-z_{1 i+1}^{[H]} \\
= & z_{1}\left(t_{i}\right)+H_{i} \Delta_{f_{1}}\left(z_{1}\left(t_{i}\right), Y_{21}\left(t_{i}\right), t_{i}, H_{i}\right)-\left(z_{1 i}+H_{i} \psi_{f_{1}}\left(z_{1 i}, \ldots, z_{1 i-n}, \tilde{Y}_{21 i+1}, \ldots, \tilde{Y}_{21 i-n}, t_{i}, H_{i}\right)\right) \\
= & H_{i}\left(\Delta_{f_{1}}\left(z_{1}\left(t_{i}\right), Y_{21}\left(t_{i}\right), t_{i}, H_{i}\right)-\psi_{f_{1}}\left(z_{1 i}, \ldots, z_{1 i-n}, Y_{21}\left(t_{i+1}\right), \ldots, Y_{21}\left(t_{i-n}\right), t_{i}, H_{i}\right)\right) \\
& +H_{i}\left(\psi_{f_{1}}\left(z_{1 i}, \ldots, z_{1 i-n}, Y_{21}\left(t_{i+1}\right), \ldots, Y_{21}\left(t_{i-n}\right), t_{i}, H_{i}\right)\right. \\
& \left.-\psi_{f_{1}}\left(z_{1 i}, \ldots, z_{1 i-n}, \tilde{Y}_{21 i+1}, \ldots, \tilde{Y}_{21 i-n}, t_{i}, H_{i}\right)\right) \\
= & H_{i}\left(\Delta_{f_{1}}\left(z_{1}\left(t_{i}\right), Y_{21}\left(t_{i}\right), t_{i}, H_{i}\right)-\psi_{f_{1}}\left(z_{1 i}, \ldots, z_{1 i-n}, Y_{21}\left(t_{i+1}\right), \ldots, Y_{21}\left(t_{i-n}\right), t_{i}, H_{i}\right)\right) \\
& +\left.H_{i} \frac{\partial \psi_{f 1}}{\partial Y_{21 i+1}}\right|_{\tilde{Y}_{21 i+1}}\left(Y_{21}\left(t_{i+1}\right)-\tilde{Y}_{21 i+1}\right)+H_{i} \cdot O\left(\left(Y_{21}\left(t_{i+1}\right)-\tilde{Y}_{21 i+1}\right)^{2}\right) .
\end{aligned}
$$

Denote

$$
\begin{aligned}
& \varepsilon_{\psi f_{1}}^{[H]}=H_{i}\left(\Delta_{f_{1}}\left(z_{1}\left(t_{i}\right), Y_{21}\left(t_{i}\right), t_{i}, H_{i}\right)-\psi_{f_{1}}\left(z_{1 i}, \ldots, z_{1 i-n}, Y_{21}\left(t_{i+1}\right), \ldots, Y_{21}\left(t_{i-n}\right), t_{i}, H_{i}\right)\right), \\
& \varepsilon_{\text {int } 2[H]}^{[H]}=\left.H_{i} \frac{\partial \psi_{f_{1}}}{\partial Y_{21 i+1}}\right|_{\tilde{Y}_{21 i+1}}\left(Y_{21}\left(t_{i+1}\right)-\tilde{Y}_{21 i+1}\right)+H_{i} \cdot O\left(\left(Y_{21}\left(t_{i+1}\right)-\tilde{Y}_{21 i+1}\right)^{2}\right) .
\end{aligned}
$$

Using (11), we can get

$$
\varepsilon_{1 i+1}^{[H]}=\varepsilon_{\psi f_{1}}^{[H]}+\varepsilon_{\text {int2 }}^{[H]} .
$$

The local truncation error in (12) of the subsystem $S_{1}$ includes two parts: the cumulative integration error $\varepsilon_{\psi f_{1}}$ caused by the integration algorithm for $\mathrm{S}_{1}$, and the interpolation error $\varepsilon_{\mathrm{int} 2}$ of the input vector $\tilde{Y}_{21 i}$ caused by transferring from subsystem $\mathrm{S}_{2}$ to subsystem $\mathrm{S}_{1}$.

\subsection{Integration error}

From the Richardson extrapolation in (4), the calculation error caused by the integration algorithm for subsystem $\mathrm{S}_{1}$ by the collaboration simulation step $H_{i}$ can be calculated as follows:

$$
\begin{aligned}
\varepsilon_{\psi f_{1}}^{[H]} & =H_{i}\left(\Delta_{f_{1}}\left(z_{1}\left(t_{i}\right), Y_{21}\left(t_{i}\right), t_{i}, H_{i}\right)-\psi_{f_{1}}\left(z_{1 i}, \ldots, z_{1 i-n}, Y_{21}\left(t_{i+1}\right), \ldots, Y_{21}\left(t_{i-n}\right), t_{i}, H_{i}\right)\right) \\
& =z_{1}\left(t_{i+1}\right)-z_{1}\left(t_{i}\right)-H_{i} \psi_{f_{1}}\left(z_{1 i}, \ldots, z_{1 i-n}, Y_{21}\left(t_{i+1}\right), \ldots, Y_{21}\left(t_{i-n}\right), t_{i}, H_{i}\right) \\
& =c_{\psi f_{1}}^{\left(p_{1}+1\right)} z_{1 i}^{\left(p_{1}+1\right)} H_{i}^{p_{1}+1}+O\left(H_{i}^{p_{1}+2}\right) \\
& =C_{1}\left(t_{i}\right) H_{i}^{p_{1}+1}+O\left(H_{i}^{p_{1}+2}\right),
\end{aligned}
$$

where $p_{1}$ is the order of increment function $\psi_{f_{1}}$ of the integration method, $c_{\psi f_{1}}^{\left(p_{1}+1\right)}$ is the error constant determined by the order $p_{1}$ and integration method together, $z_{1 i}^{\left(p_{1}+1\right)}$ is the derivative of state vector $z_{1}$ at time $t_{i}$ with order $p_{1}+1$, and $C_{1}\left(t_{i}\right)=c_{\psi f_{1}}^{\left(p_{1}+1\right)} z_{1 i}^{\left(p_{1}+1\right)}$.

\subsection{Interpolation error}

To get the interpolation error $\varepsilon_{\text {int2 }}$ caused by the input vector $\tilde{Y}_{21}(t)=I_{Y 2 i}\left(Y_{2 i}, T_{i}\right)(t)$ from subsystem $\mathrm{S}_{2}$ to $\mathrm{S}_{1}$, assume that the coupling vector $Y_{21}(t)$ in (10) has the following property: $Y_{21}^{\left(q_{2}\right)}(t)$ is continuous in $\left[t_{i-q_{2}}, t_{i+1}\right]$ and $Y_{21}^{\left(q_{2}+1\right)}(t)$ exists in $\left(t_{i-q_{2}}, t_{i+1}\right) . I_{Y 2 i}(t)$ is a polynomial satisfying above Lagrange interpolation conditions, $q_{2}$ is the order of the interpolation formula $I_{Y 2 i}$. Denote $R(t)=Y_{21}(t)-\tilde{Y}_{21}(t)=$ $Y_{21}(t)-I_{Y 2 i}\left(Y_{2 i}, T_{i}\right)(t)$, where $t \in\left[t_{i-q_{2}}, t_{i+1}\right]$. Based on the remainder term theorem of Lagrange interpolation polynomial, we have

$$
R(t)=\left(t-t_{i}\right)\left(t-t_{i-1}\right) \cdots\left(t-t_{i-q_{2}}\right) Y_{21 \eta}^{\left(q_{2}+1\right)} /\left(q_{2}+1\right) !=\omega_{q_{2}+1}(t) \cdot Y_{21 \eta}^{\left(q_{2}+1\right)} /\left(q_{2}+1\right) !
$$


where $\omega_{q_{2}+1}\left(t_{i+1}\right)=\left(t_{i+1}-t_{i}\right)\left(t_{i+1}-t_{i-1}\right) \cdots\left(t_{i+1}-t_{i-q_{2}}\right)=H_{i}\left(H_{i}+H_{i-1}\right) \cdots\left(H_{i}+H_{i-1}+\cdots+\right.$ $\left.H_{i-q_{2}}\right), Y_{21 \eta}^{\left(q_{2}+1\right)}$ is the $\left(q_{2}+1\right)$ th order derivative of output vector $Y_{21}(t)$ at $t_{\eta}$. So, we have

$$
\begin{aligned}
\left.H_{i} \frac{\partial \psi_{f_{1}}}{\partial Y_{21 i+1}}\right|_{\tilde{Y}_{21 i+1}}\left(Y_{21}\left(t_{i+1}\right)-\tilde{Y}_{21 i+1}\right) \\
\quad=\left.H_{i} \frac{\partial \psi_{f_{1}}}{\partial Y_{21 i+1}}\right|_{\tilde{Y}_{21 i+1}} H_{i}\left(H_{i}+H_{i-1}\right) \cdots\left(H_{i}+H_{i-1}+\cdots+H_{i-q_{2}}\right) Y_{21}^{\left(q_{2}+1\right)}\left(t_{\eta}\right) /\left(q_{2}+1\right) ! \\
\quad=\left(\left.\frac{\partial \psi_{f_{1}}}{\partial Y_{21 i+1}}\right|_{\tilde{Y}_{21 i+1}} \frac{Y_{21 \eta}^{\left(q_{2}+1\right)}}{\left(q_{2}+1\right) !}\left(1+\frac{1}{S_{i}}\right) \cdots\left(1+\frac{1}{S_{i}}+\cdots+\frac{1}{S_{i} \cdots S_{i-q_{2}+1}}\right)\right) H_{i}^{q_{2}+2} \\
\quad=C_{2}\left(t_{i+1}, H_{i}, H_{i-1}, H_{i-2}, \ldots\right) H_{i}^{q_{2}+2} .
\end{aligned}
$$

The interpolation error $\varepsilon_{\text {int2 }}^{[H]}$ can be represented as follows:

$$
\begin{aligned}
\varepsilon_{\text {int } 2}^{[H]} & =\left.H_{i} \frac{\partial \psi_{f_{1}}}{\partial Y_{21 i+1}}\right|_{\tilde{Y}_{21 i+1}}\left(Y_{21}\left(t_{i+1}\right)-\tilde{Y}_{21 i+1}\right)+H_{i} \cdot O\left(\left(Y_{21}\left(t_{i+1}\right)-\tilde{Y}_{21 i+1}\right)^{2}\right) \\
& =C_{2}\left(t_{i+1}, H_{i}, H_{i-1}, H_{i-2}, \ldots\right) H_{i}^{q_{2}+2}+O\left(H_{i}^{2 q_{2}+3}\right),
\end{aligned}
$$

where $S_{i}=\frac{H_{i}}{H_{i-1}}, \ldots, S_{i-q_{2}+1}=\frac{H_{i-q_{2}+1}}{H_{i-q_{2}}}$. These variables denote the ratio of the simulation step $H_{i}$ at a specific time point to the simulation step $H_{i-1}$ at previous time points, and their coefficients are known.

\subsection{Local truncation error at step $H_{i}$}

With formulas (13) and (14), the local truncation error in subsystem $\mathrm{S}_{1}$ can be represented as below:

$$
\begin{aligned}
\varepsilon_{1 i+1}^{[H]} & =\varepsilon_{\psi f_{1}}^{[H]}+\varepsilon_{\text {int2 }}^{[H]} \\
& =C_{1}\left(t_{i}\right) H_{i}^{p_{1}+1}+C_{2}\left(t_{i+1}, H_{i}, H_{i-1}, H_{i-2}, \ldots\right) H_{i}^{q_{2}+2}+O\left(H_{i}^{p_{1}+2}\right)+O\left(H_{i}^{2 q_{2}+3}\right) . \\
z_{1}\left(t_{i+1}\right) & =z_{1 i+1}^{[H]}+\varepsilon_{1 i+1}^{[H]} \\
& =z_{1 i+1}^{[H]}+C_{1}\left(t_{i}\right) H_{i}^{p_{1}+1}+C_{2}\left(t_{i+1}, H_{i}, H_{i-1}, H_{i-2}, \ldots\right) H_{i}^{q_{2}+2}+O\left(H_{i}^{p_{1}+2}\right)+O\left(H_{i}^{2 q_{2}+3}\right) .
\end{aligned}
$$

\section{Derivation of local truncation error}

\subsection{A two-step calculation method}

According to the Richardson extrapolation method, the calculation of (16) goes as follows: calculating the value of $z_{1 i+1}^{[H / 2]}$ at the point $t_{i}+H_{i}$ by calculating two smaller steps with a step $H_{i} / 2$, i.e.

$$
\begin{aligned}
z_{1 i+\frac{1}{2}}^{[H / 2]}= & z_{1 i}+\frac{H_{i}}{2} \psi_{f_{1}}\left(z_{1 i}, \ldots, z_{1 i-n}, \tilde{Y}_{21 i+\frac{1}{2}}, \tilde{Y}_{21 i}, \ldots, \tilde{Y}_{21 i-n}, t_{i}, \frac{H_{i}}{2}\right) . \\
z_{1 i+1}^{[H / 2]}= & z_{1 i}+\frac{H_{i}}{2} \psi_{f_{1}}\left(z_{1 i}, \ldots, z_{1 i-n}, \tilde{Y}_{21 i+\frac{1}{2}}, \tilde{Y}_{21 i}, \ldots, \tilde{Y}_{21 i-n}, t_{i}, \frac{H_{i}}{2}\right) \\
& +\frac{H_{i}}{2} \psi_{f_{1}}\left(z_{1 i+\frac{1}{2}}^{[H / 2]}, z_{1 i}, \ldots, z_{1 i-n+1}, \tilde{Y}_{21 i+1}, Y_{21 i+\frac{1}{2}}, \tilde{Y}_{21 i}, \ldots, \tilde{Y}_{21 i-n+1}, t_{i+\frac{1}{2}}, \frac{H_{i}}{2}\right),
\end{aligned}
$$

where $z_{1 i}=z_{1}\left(t_{i}\right), z_{1 i-1}=z_{1}\left(t_{i-1}\right), \ldots . Y_{21 i+\frac{1}{2}}(t)$ can be calculated either by using the extrapolation function $\tilde{Y}_{21 i+\frac{1}{2}}(t)$ or by using $Y_{21 i+\frac{1}{2}}^{[H / 2]}(t)$, calculated by subsystem $\mathrm{S}_{2}$ after integration calculation with the first step $H_{i} / 2 ; \tilde{Y}_{21 i+1}(t)$ is an extrapolation function constructed by $Y_{21 i+\frac{1}{2}}(t), Y_{21}\left(t_{i}\right) ; Y_{21}\left(t_{i-1}\right), \ldots$ So, we have

$$
\begin{aligned}
\varepsilon_{1 i+1}^{[H / 2]} & =z_{1}\left(t_{i+1}\right)-z_{1 i+1}^{[H / 2]} \\
& =z_{1}\left(t_{i}\right)+\frac{H_{i}}{2} \Delta_{f_{1}}\left(z_{1}\left(t_{i}\right), Y_{21}\left(t_{i}\right), t_{i}, \frac{H_{i}}{2}\right)+\frac{H_{i}}{2} \Delta_{f_{1}}\left(z_{1}\left(t_{i+\frac{1}{2}}\right), Y_{21}\left(t_{i+\frac{1}{2}}\right), t_{i+\frac{1}{2}}, \frac{H_{i}}{2}\right)
\end{aligned}
$$




$$
\begin{aligned}
& -\left(z_{1 i}+\frac{H_{i}}{2} \psi_{f_{1}}\left(z_{1 i}, \ldots, z_{1 i-n}, \tilde{Y}_{21 i+\frac{1}{2}}, \tilde{Y}_{21 i}, \ldots, \tilde{Y}_{21 i-n}, t_{i}, \frac{H_{i}}{2}\right)\right. \\
& \left.+\frac{H_{i}}{2} \psi_{f_{1}}\left(z_{1 i+\frac{1}{2}}^{[H / 2]}, z_{1 i}, \ldots, z_{1 i-n+1}, \tilde{Y}_{21 i+1}, Y_{21 i+\frac{1}{2}}, \tilde{Y}_{21 i}, \ldots, \tilde{Y}_{21 i-n+1}, t_{i+\frac{1}{2}}, \frac{H_{i}}{2}\right)\right) \\
& =\frac{H_{i}}{2}\left(\Delta_{f_{1}}\left(z_{1}\left(t_{i}\right), Y_{21}\left(t_{i}\right), t_{i}, \frac{H_{i}}{2}\right)-\psi_{f_{1}}\left(z_{1 i}, \ldots, z_{1 i-n}, \tilde{Y}_{21 i+\frac{1}{2}}, \tilde{Y}_{21 i}, \ldots, \tilde{Y}_{21 i-n}, t_{i}, \frac{H_{i}}{2}\right)\right) \\
& +\frac{H_{i}}{2}\left(\Delta_{f_{1}}\left(z_{1}\left(t_{i+\frac{1}{2}}\right), Y_{21}\left(t_{i+\frac{1}{2}}\right), t_{i+\frac{1}{2}}, \frac{H_{i}}{2}\right)\right. \\
& \left.-\psi_{f_{1}}\left(z_{1 i+\frac{1}{2}}^{[H / 2]}, z_{1 i}, \ldots, z_{1 i-n+1}, \tilde{Y}_{21 i+1}, Y_{21 i+\frac{1}{2}}, \tilde{Y}_{21 i}, \ldots, \tilde{Y}_{21 i-n+1}, t_{i+\frac{1}{2}}, \frac{H_{i}}{2}\right)\right) \\
& =\frac{H_{i}}{2}\left(\Delta_{f_{1}}\left(z_{1}\left(t_{i}\right), Y_{21}\left(t_{i}\right), t_{i}, \frac{H_{i}}{2}\right)-\psi_{f_{1}}\left(z_{1 i}, \ldots, z_{1 i-n}, Y_{21}\left(t_{i+\frac{1}{2}}\right), Y_{21}\left(t_{i}\right), \ldots, Y_{21}\left(t_{i-n}\right), t_{i}, \frac{H_{i}}{2}\right)\right) \\
& +\frac{H_{i}}{2}\left(\psi_{f_{1}}\left(z_{1 i}, \ldots, z_{1 i-n}, Y_{21}\left(t_{i+\frac{1}{2}}\right), Y_{21}\left(t_{i}\right), \ldots, Y_{21}\left(t_{i-n}\right), t_{i}, \frac{H_{i}}{2}\right)\right. \\
& \left.-\psi_{f_{1}}\left(z_{1 i}, \ldots, z_{1 i-n}, \tilde{Y}_{21 i+\frac{1}{2}}, \tilde{Y}_{21 i}, \ldots, \tilde{Y}_{21 i-n}, t_{i}, \frac{H_{i}}{2}\right)\right) \\
& +\frac{H_{i}}{2}\left(\Delta_{f_{1}}\left(z_{1}\left(t_{i+\frac{1}{2}}\right), Y_{21}\left(t_{i+\frac{1}{2}}\right), t_{i+\frac{1}{2}}, \frac{H_{i}}{2}\right)\right. \\
& \left.-\psi_{f_{1}}\left(z_{1 i+\frac{1}{2}}, z_{1 i}, \ldots, z_{1 i-n+1}, Y_{21}\left(t_{i+1}\right), Y_{21}\left(t_{i+\frac{1}{2}}\right), Y_{21}\left(t_{i}\right), \ldots, Y_{21}\left(t_{i-n+1}\right), t_{i}, \frac{H_{i}}{2}\right)\right) \\
& +\frac{H_{i}}{2}\left(\begin{array}{l}
\psi_{f_{1}}\left(z_{1 i+\frac{1}{2}}, z_{1 i}, \ldots, z_{1 i-n+1}, Y_{21}\left(t_{i+1}\right), Y_{21}\left(t_{i+\frac{1}{2}}\right), Y_{21}\left(t_{i}\right), \ldots, Y_{21}\left(t_{i-n+1}\right), t_{i}, \frac{H_{i}}{2}\right) \\
-\psi_{f_{1}}\left(z_{1 i+\frac{1}{2}}^{[H / 2]}, z_{1 i}, \ldots, z_{1 i-n+1}, \tilde{Y}_{21 i+1}, Y_{21 i+\frac{1}{2}}, \tilde{Y}_{21 i}, \ldots, \tilde{Y}_{21 i-n+1}, t_{i+\frac{1}{2}}, \frac{H_{i}}{2}\right)
\end{array}\right) \\
& =C_{1}\left(t_{i}\right)\left(\frac{H_{i}}{2}\right)^{p_{1}+1}+O\left(H_{i}^{p_{1}+2}\right)+C_{2}\left(t_{i+\frac{1}{2}}, \frac{H_{i}}{2}, H_{i-1}, H_{i-2}, \ldots\right)\left(\frac{H_{i}}{2}\right)^{q_{2}+2}+O\left(H_{i}^{2 q_{2}+3}\right) \\
& +C_{1}\left(t_{i+\frac{1}{2}}\right)\left(\frac{H_{i}}{2}\right)^{p_{1}+1}+O\left(H_{i}^{p_{1}+2}\right)+\left(O\left(H_{i}^{p_{1}+2}\right)+O\left(H_{i}^{q_{2}+3}\right)\right) \\
& +C_{2}\left(t_{i+1}, \frac{H_{i}}{2}, \frac{H_{i}}{2}, H_{i-1}, \ldots\right)\left(\frac{H_{i}}{2}\right)^{q_{2}+2}+O\left(H_{i}^{2 q_{2}+3}\right) \\
& +\left(O\left(H_{i}^{p_{1}+2}\right)+O\left(H_{i}^{q_{2}+3}\right)\right)+\left(O\left(H_{i}^{p_{1}+2}\right)+O\left(H_{i}^{q_{2}+3}\right)\right) .
\end{aligned}
$$

Using Taylor expansion, the calculation procedures can be analyzed as follows.

Firstly, in (18), replacing $z_{1 i+\frac{1}{2}}^{[H / 2]}$ by the approximation value $z_{1}\left(t_{i+\frac{1}{2}}\right)$, the error for approximation is $\left(O\left(H_{i}^{p_{1}+2}\right)+O\left(H_{i}^{q_{2}+3}\right)\right)$.

Secondly, replacing $Y_{21 i+\frac{1}{2}}$ by the approximation value $Y_{21}\left(t_{i+\frac{1}{2}}\right)$, the error for approximation is $\left(O\left(H_{i}^{p_{1}+2}\right)+O\left(H_{i}^{q_{2}+3}\right)\right)$, provided all of the following three conditions are satisfied.

a) $Y_{21 i+\frac{1}{2}}(t)$ is assigned as the value $Y_{21 i+\frac{1}{2}}^{[H / 2]}(t)$, which is the numerical solution of $\mathrm{S}_{2}$, i.e. two subsystems exchange endpoints data after integration calculation with the first step $H_{i} / 2$.

b) The progress of integration calculation with the first step $H_{i} / 2$ is successive as in [17]. For example, subsystem $\mathrm{S}_{1}$ goes progressively with step $H_{i} / 2$ by using extrapolation function, and then subsystem $\mathrm{S}_{2}$ goes progressively with step $H_{i} / 2$ by using interpolation calculation, which takes the final output of $\mathrm{S}_{1}$ as its input.

c) The system satisfies the stability condition in [20]; that is, algebraic loops do not exist between the outputs in (9), (10), i.e. there exists at least one subsystem, whose output does not depend on the input from another subsystem.

Thirdly, the next approximation can be made as follows: replacing the extrapolation function $\tilde{Y}_{21 i+1}$ by using estimation values $Y_{21 i+\frac{1}{2}}, Y_{21}\left(t_{i}\right), Y_{21}\left(t_{i-1}\right), \ldots$ with $\tilde{Y}_{21 i+1}$ by precise values $Y_{21}\left(t_{i+\frac{1}{2}}\right), Y_{21}\left(t_{i}\right)$, $Y_{21}\left(t_{i-1}\right), \ldots$ The error for this approximation is $O\left(H_{i}^{p_{1}+2}\right)+O\left(H_{i}^{q_{2}+3}\right)$. 
Lastly, $C_{1}\left(t_{i+\frac{1}{2}}\right)$ can be approximated as $C_{1}\left(t_{i}\right)$ with an error $O\left(H_{i}^{p_{1}+2}\right)$. Then (18) can be represented as

$$
\begin{aligned}
\varepsilon_{1 i+1}^{[H / 2]}= & 2 C_{1}\left(t_{i}\right)\left(\frac{H_{i}}{2}\right)^{p_{1}+1}+\left(C_{2}\left(t_{i+\frac{1}{2}}, \frac{H_{i}}{2}, H_{i-1}, H_{i-2}, \ldots\right)\right. \\
& \left.+C_{2}\left(t_{i+1}, \frac{H_{i}}{2}, \frac{H_{i}}{2}, H_{i-1}, \ldots\right)\right)\left(\frac{H_{i}}{2}\right)^{q_{2}+2}+O\left(H_{i}^{p_{1}+2}\right)+O\left(H_{i}^{q_{2}+3}\right) .
\end{aligned}
$$

So, we get

$$
\begin{aligned}
z_{1}\left(t_{i+1}\right)= & z_{1 i+1}^{[H / 2]}+\varepsilon_{1 i+1}^{[H / 2]} \\
= & z_{1 i+1}^{[H / 2]}+2 C_{1}\left(t_{i}\right)\left(\frac{H_{i}}{2}\right)^{p_{1}+1}+\left(C_{2}\left(t_{i+\frac{1}{2}}, \frac{H_{i}}{2}, H_{i-1}, H_{i-2}, \ldots\right)\right. \\
& \left.+C_{2}\left(t_{i+1}, \frac{H_{i}}{2}, \frac{H_{i}}{2}, H_{i-1}, \ldots\right)\right)\left(\frac{H_{i}}{2}\right)^{q_{2}+2}+O\left(H_{i}^{p_{1}+2}\right)+O\left(H_{i}^{q_{2}+3}\right) .
\end{aligned}
$$

To analyze terms $C_{2}\left(t_{i+\frac{1}{2}}, \frac{H_{i}}{2}, H_{i-1}, H_{i-2}, \ldots\right)$ and $C_{2}\left(t_{i+1}, \frac{H_{i}}{2}, \frac{H_{i}}{2}, H_{i-1}, \ldots\right)$ in (18), letting $S_{i}^{\prime}=$ $\frac{H_{i}}{2} / H_{i-1}=\frac{S_{i}}{2}, S_{i}^{\prime \prime}=\frac{H_{i}}{2} / \frac{H_{i}}{2}=1$, we have

$$
\begin{aligned}
& \psi_{f_{1}}\left(z_{1 i}, \ldots, z_{1 i-n}, Y_{21}\left(t_{i+\frac{1}{2}}\right), Y_{21}\left(t_{i}\right), \ldots, Y_{21}\left(t_{i-n}\right), t_{i}, \frac{H_{i}}{2}\right) \\
& -\psi_{f_{1}}\left(z_{1 i}, \ldots, z_{1 i-n}, \tilde{Y}_{21 i+\frac{1}{2}}, \tilde{Y}_{21 i}, \ldots, \tilde{Y}_{21 i-n}, t_{i}, \frac{H_{i}}{2}\right) \\
= & \left.\frac{\partial \psi_{f_{1}}}{\partial Y_{21 i+1}}\right|_{\tilde{Y}_{21 i+\frac{1}{2}}}\left(Y_{21}\left(t_{i+\frac{1}{2}}\right)-\tilde{Y}_{21 i+\frac{1}{2}}\right) \\
= & \left.\frac{\partial \psi_{f_{1}}}{\partial Y_{21 i+1}}\right|_{\tilde{Y}_{21 i+\frac{1}{2}}} \frac{H_{i}}{2}\left(\frac{H_{i}}{2}+H_{i-1}\right) \cdots\left(\frac{H_{i}}{2}+H_{i-1}+\cdots+H_{i-q_{2}}\right) Y_{21}^{\left(q_{2}+1\right)}\left(t_{\eta^{\prime}}\right) /\left(q_{2}+1\right) ! \\
= & \left(\left.\frac{\partial \psi_{f_{1}}}{\partial Y_{21 i+1}}\right|_{\tilde{Y}_{21 i+\frac{1}{2}}} \frac{Y_{21 \eta^{\prime}}^{\left(q_{2}+1\right)}}{\left(q_{2}+1\right) !}\left(1+\frac{1}{S_{i}^{\prime}}\right) \cdots\left(1+\frac{1}{S_{i}^{\prime}}+\frac{1}{S_{i}^{\prime} S_{i-1}}+\cdots+\frac{1}{S_{i}^{\prime} S_{i-1} \cdots S_{i-q_{2}+1}}\right)\right) \\
= & C_{2}\left(t_{i+\frac{1}{2}}, \frac{H_{i}}{2}, H_{i-1}, H_{i-2}, \ldots\right)\left(\frac{H_{i}}{2}\right)^{q_{2}+1} .
\end{aligned}
$$

In addition, we have

$$
\begin{aligned}
& \psi_{f_{1}}\left(z_{1 i+\frac{1}{2}}, z_{1 i}, \ldots, z_{1 i-n+1}, Y_{21}\left(t_{i+1}\right), Y_{21}\left(t_{i+\frac{1}{2}}\right), Y_{21}\left(t_{i}\right), \ldots, Y_{21}\left(t_{i-n+1}\right), t_{i}, \frac{H_{i}}{2}\right) \\
& -\psi_{f_{1}}\left(z_{1 i+\frac{1}{2}}, z_{1 i}, \ldots, z_{1 i-n+1}, \tilde{Y}_{21 i+1}, \tilde{Y}_{21 i+\frac{1}{2}}, \tilde{Y}_{21 i}, \ldots, \tilde{Y}_{21 i-n+1}, t_{i+\frac{1}{2}}, \frac{H_{i}}{2}\right) \\
= & \left.\frac{\partial \psi_{f_{1}}}{\partial Y_{21 i+1}}\right|_{\tilde{Y}_{21 i+1}}\left(Y_{21}\left(t_{i+1}\right)-\tilde{Y}_{21 i+1}\right) \\
= & \left.\frac{\partial \psi_{f_{1}}}{\partial Y_{21 i+1}}\right|_{\tilde{Y}_{21 i+1}} \frac{H_{i}}{2}\left(\frac{H_{i}}{2}+\frac{H_{i}}{2}\right)\left(\frac{H_{i}}{2}+\frac{H_{i}}{2}+H_{i-1}\right) \\
& \cdots\left(\frac{H_{i}}{2}+\frac{H_{i}}{2}+H_{i-1}+\cdots+H_{i-q_{2}+1}\right) Y_{21}^{\left(q_{2}+1\right)}\left(t_{\eta^{\prime \prime}}\right) /\left(q_{2}+1\right) ! \\
= & \left(\left.\frac{\partial \psi_{f_{1}}}{\partial Y_{21 i+1}}\right|_{\tilde{Y}_{21 i+1}} \frac{Y_{21 \eta^{\prime \prime}}^{\left(q_{2}+1\right)}}{\left(q_{2}+1\right) !}\left(1+\frac{1}{S_{i}^{\prime \prime}}\right)\left(1+\frac{1}{S_{i}^{\prime \prime}}+\frac{1}{S_{i}^{\prime}}\right) \cdots\left(1+\frac{1}{S_{i}^{\prime \prime}}+\frac{1}{S_{i}^{\prime}}+\cdots+\frac{1}{S_{i}^{\prime} S_{i-1} \cdots S_{i-q_{2}+2}}\right)\right) \\
= & C_{2}\left(t_{i+1}, \frac{H_{i}}{2}, \frac{H_{i}}{2}, H_{i-1}, \ldots\right)\left(\frac{H_{i}}{2}\right)^{q_{2}+1},
\end{aligned}
$$

where $\tilde{Y}_{21 i+\frac{1}{2}}=Y_{21}\left(t_{i+\frac{1}{2}}\right), \tilde{Y}_{21 i}=Y_{21}\left(t_{i}\right), \ldots$, and $\tilde{Y}_{21 i+1}(t)$ is the extrapolation function constructed by 
using $Y_{21}\left(t_{i+\frac{1}{2}}\right), Y_{21}\left(t_{i}\right), Y_{21}\left(t_{i-1}\right), \ldots$, and letting

$$
\begin{aligned}
& S_{a}=\frac{C_{2}\left(t_{i+\frac{1}{2}}, \frac{H_{i}}{2}, H_{i-1}, H_{i-2}, \ldots\right)}{C_{2}\left(t_{i+1}, H_{i}, H_{i-1}, H_{i-2}, \ldots\right)} \approx \frac{\left(1+\frac{1}{S_{i}^{\prime}}\right) \cdots\left(1+\frac{1}{S_{i}^{\prime}}+\frac{1}{S_{i}^{\prime} S_{i-1}} \cdots+\frac{1}{S_{i}^{\prime} S_{i-1} \cdots S_{i-q_{2}+1}}\right)}{\left(1+\frac{1}{S_{i}}\right) \cdots\left(1+\frac{1}{S_{i}}+\cdots+\frac{1}{S_{i} \cdots S_{i-q_{2}+1}}\right)}, \\
& S_{b}=\frac{C_{2}\left(t_{i+1}, \frac{H_{i}}{2}, \frac{H_{i}}{2}, H_{i-1}, \ldots\right)}{C_{2}\left(t_{i+1}, H_{i}, H_{i-1}, H_{i-2}, \ldots\right)} \approx \frac{\left(1+\frac{1}{S_{i}^{\prime \prime}}\right)\left(1+\frac{1}{S_{i}^{\prime \prime}}+\frac{1}{S_{i}^{\prime}}\right) \cdots\left(1+\frac{1}{S_{i}^{\prime \prime}}+\frac{1}{S_{i}^{\prime}} \cdots+\frac{1}{S_{i}^{\prime} S_{i-1} \cdots S_{i-q_{2}+2}}\right)}{\left(1+\frac{1}{S_{i}}\right) \cdots\left(1+\frac{1}{S_{i}}+\cdots+\frac{1}{S_{i} \cdots S_{i-q_{2}+1}}\right)} .
\end{aligned}
$$

In above formulas $S_{a}$ and $S_{b}$, replacing $\eta^{\prime}$ and $\eta^{\prime \prime}$ by the approximation value $\eta$, and replacing $\left.\frac{\partial \psi_{f 1}}{\partial Y_{21 i+1}}\right|_{\tilde{Y}_{21 i+\frac{1}{2}}}$ by the approximated value $\left.\frac{\partial \psi_{f 1}}{\partial Y_{21 i+1}}\right|_{\tilde{Y}_{21 i+1}}$, from (16) and (19), we have

$$
\left\{\begin{aligned}
\varepsilon_{1 i+1}^{[H]}= & z_{1}\left(t_{i+1}\right)-z_{1 i+1}^{[H]}=C_{1}\left(t_{i}\right) H_{i}^{p_{1}+1}+C_{2}\left(t_{i+1}, H_{i}, H_{i-1}, H_{i-2}, \ldots\right) H_{i}^{q_{2}+2} \\
& +O\left(H_{i}^{p_{1}+2}\right)+O\left(H_{i}^{2 q_{2}+3}\right), \\
\varepsilon_{1 i+1}^{[H / 2]}= & z_{1}\left(t_{i+1}\right)-z_{1 i+1}^{[H / 2]}=2 C_{1}\left(t_{i}\right)\left(\frac{H_{i}}{2}\right)^{p_{1}+1}+\left(S_{a}+S_{b}\right) C_{2}\left(t_{i+1}, H_{i},\right. \\
& \left.H_{i-1}, H_{i-2}, \ldots\right)\left(\frac{H_{i}}{2}\right)^{q_{2}+2}+O\left(H_{i}^{p_{1}+2}\right)+O\left(H_{i}^{q_{2}+3}\right) .
\end{aligned}\right.
$$

where $\varepsilon_{1 i+1}^{[H / 2]}$ is yielded by using Taylor expansion. Considering the above approximations in $S_{a}$ and $S_{b}$, the errors for both approximations in $(20)$ are $O\left(H_{i}^{q_{2}+3}\right)$.

\section{$5.2 \quad$ Local truncation error}

$C_{1}\left(t_{i}\right)$ and $C_{2}\left(t_{i+1}, H_{i}, H_{i-1}, H_{i-2}, \ldots\right)$ can be calculated by using (20). From (20), we can also obtain the estimation expression of the local truncation error for subsystem $S_{1}$ in (9). This can be discussed in the following cases:

1) When $p_{1}<q_{2}+1$ such that $C_{2}\left(t_{i+1}, H_{i}, H_{i-1}, H_{i-2}, \ldots\right) H_{i}^{q_{2}+2}=O\left(H_{i}^{p_{1}+2}\right)$, the errors caused by the interpolations of the coupling vectors can be omitted; thus we get

$$
C_{1}\left(t_{i}\right) \approx \frac{1}{H_{i}^{p_{1}+1}\left(1-2^{-p_{1}}\right)}\left(z_{1 i+1}^{[H / 2]}-z_{1 i+1}^{[H]}\right) .
$$

Then, estimation formula of the local truncation error for subsystem $\mathrm{S}_{1}$ is as follows:

$$
\left\{\begin{array}{l}
\varepsilon_{1 i+1}^{[H]} \approx \frac{1}{\left(1-2^{\left.-p_{1}\right)}\right.}\left(z_{1 i+1}^{[H / 2]}-z_{1 i+1}^{[H]}\right), \\
\varepsilon_{1 i+1}^{[H / 2]} \approx \frac{1}{\left(2^{p_{1}}-1\right)}\left(z_{1 i+1}^{[H / 2]}-z_{1 i+1}^{[H]}\right) .
\end{array}\right.
$$

2) When $p_{1}>q_{2}+1$ such that $C_{1}\left(t_{i}\right) H_{i}^{p_{1}+1}=O\left(H_{i}^{q_{2}+3}\right)$, this term can be omitted. And the local truncation error of subsystem $S_{1}$ is mainly dominated by the interpolation error caused by the coupling vector $\tilde{Y}_{21}(t)$. So, we get

$$
C_{2}\left(t_{i+1}, H_{i}, H_{i-1}, H_{i-2}, \ldots\right) \approx \frac{1}{H_{i}^{q_{2}+2}\left(1-\left(S_{a}+S_{b}\right) 2^{-\left(q_{2}+2\right)}\right)}\left(z_{1 i+1}^{[H / 2]}-z_{1 i+1}^{[H]}\right) .
$$

Then the estimation formula of local truncation error for subsystem $S_{1}$ is as follows:

$$
\left\{\begin{array}{l}
\varepsilon_{1 i+1}^{[H]} \approx \frac{1}{\left(1-\left(S_{a}+S_{b}\right) 2^{-\left(q_{2}+2\right)}\right)}\left(z_{1 i+1}^{[H / 2]}-z_{1 i+1}^{[H]}\right), \\
\varepsilon_{1 i+1}^{[H / 2]} \approx \frac{1}{\left(\left(S_{a}+S_{b}\right)^{-1} 2^{q_{2}+2}-1\right)}\left(z_{1 i+1}^{[H / 2]}-z_{1 i+1}^{[H]}\right) .
\end{array}\right.
$$

3) When $p_{1}=q_{2}+1$ with $S_{a}+S_{b}=2$ (this condition can be permitted if using 0-order interpolation method and 1-order integration calculation, i.e., when $q_{2}=0, S_{a}=1, S_{b}=1$ ), we have

$$
C_{1}\left(t_{i}\right)+C_{2}\left(t_{i+1}, H_{i}, H_{i-1}, H_{i-2}, \ldots\right) \approx \frac{1}{H_{i}^{p_{1}+1}\left(1-2^{-p_{1}}\right)}\left(z_{1 i+1}^{[H / 2]}-z_{1 i+1}^{[H]}\right) .
$$


So the estimation formula of local truncation error for subsystem $S_{1}$ is as follows:

$$
\left\{\begin{array}{l}
\varepsilon_{1 i+1}^{[H]} \approx \frac{1}{\left(1-2^{-p_{1}}\right)}\left(z_{1 i+1}^{[H / 2]}-z_{1 i+1}^{[H]}\right), \\
\varepsilon_{1 i+1}^{[H / 2]} \approx \frac{1}{\left(2^{p_{1}}-1\right)}\left(z_{1 i+1}^{[H / 2]}-z_{1 i+1}^{[H]}\right) .
\end{array}\right.
$$

4) When $p_{1}=q_{2}+1$, and $S_{a}+S_{b} \neq 2$ (this condition can be permitted if interpolation method has an order larger than 0 ), the two equations in (20) can be combined to form a linear equation with two unknowns related to $C_{1}\left(t_{i}\right)$ and $C_{2}\left(t_{i+1}, H_{i}, H_{i-1}, H_{i-2}, \ldots\right)$. So, $C_{1}\left(t_{i}\right)$ and $C_{2}\left(t_{i+1}, H_{i}, H_{i-1}, H_{i-2}, \ldots\right)$ cannot be solved. Therefore the Richardson extrapolation method needs to be extended according to one of the following two methods: one of integration calculation for (17) is also to divide the second step $H_{i} / 2$ into two step sizes $\left(H_{i} / 4, H_{i} / 4\right)$, and the other is to make integration calculation for (17) by using two step sizes $\left(3 H_{i} / 4, H_{i} / 4\right)$. Then by combining (20), the estimation formula of local truncation error for subsystem $\mathrm{S}_{1}$ can be obtained.

The above estimation formula of the local truncation error for state vector $z_{1}$ in subsystem $\mathrm{S}_{1}$ is also appropriate for state vector $z_{2}$ in subsystem $\mathrm{S}_{2}$.

\section{Simulation experiments for the evaluation}

To evaluate the validity of the proposed method, collaborative simulation is performed for a coupled system using the variable-step algorithm. The equations for the two sub-system models are shown in eqs. (27) and (28) respectively.

$$
\begin{aligned}
& \left\{\begin{array}{l}
\frac{d x_{1}}{d t}=x_{1} y_{3}-x_{2}, \\
\frac{d x_{2}}{d t}=-2 x_{1} x_{2}+y_{4} \\
y_{1}=x_{2} y_{3} \\
y_{2}=3 x_{1} x_{2}+y_{3} y_{4} .
\end{array}\right. \\
& \left\{\begin{array}{l}
\frac{d x_{3}}{d t}=3 x_{4} y_{1}+x_{3}, \\
\frac{d x_{4}}{d t}=2 x_{3} x_{4}-y_{2}, \\
y_{3}=4 x_{3}+x_{4} \\
y_{4}=x_{3}-2 x_{4} .
\end{array}\right.
\end{aligned}
$$

For the three different situations (eqs. (22), (24) and (26) respectively) discussed above for controlling the truncation error, simulations are performed by using different integration algorithms, as well different interpolation algorithms. To evaluate the performance of the simulations performed, the results obtained are compared with those obtained from simulations that utilize the interfaces between simulation tools, as well as those obtained from the simulations that utilize constant simulation step size [17]. The settings for the simulation are as follows:

Initial values: $x_{1}=-1, x_{2}=1, x_{3}=-1, x_{4}=1, y_{1}=-3, y_{2}=6, y_{3}=-3, y_{4}=-3$.

Time period: $0 \sim 2$ seconds; controlled accuracy of truncation error: 0.01 ; minimum simulation step: 0.001 second.

The simulation computations of variables and truncation errors for (27) and (28) are archived respectively. Due to the limitation of the space, only the simulation results of variable $x_{1}$ are given in the figures. The simulation results of $x_{1}$ under three different situations as above formulas of local truncation 

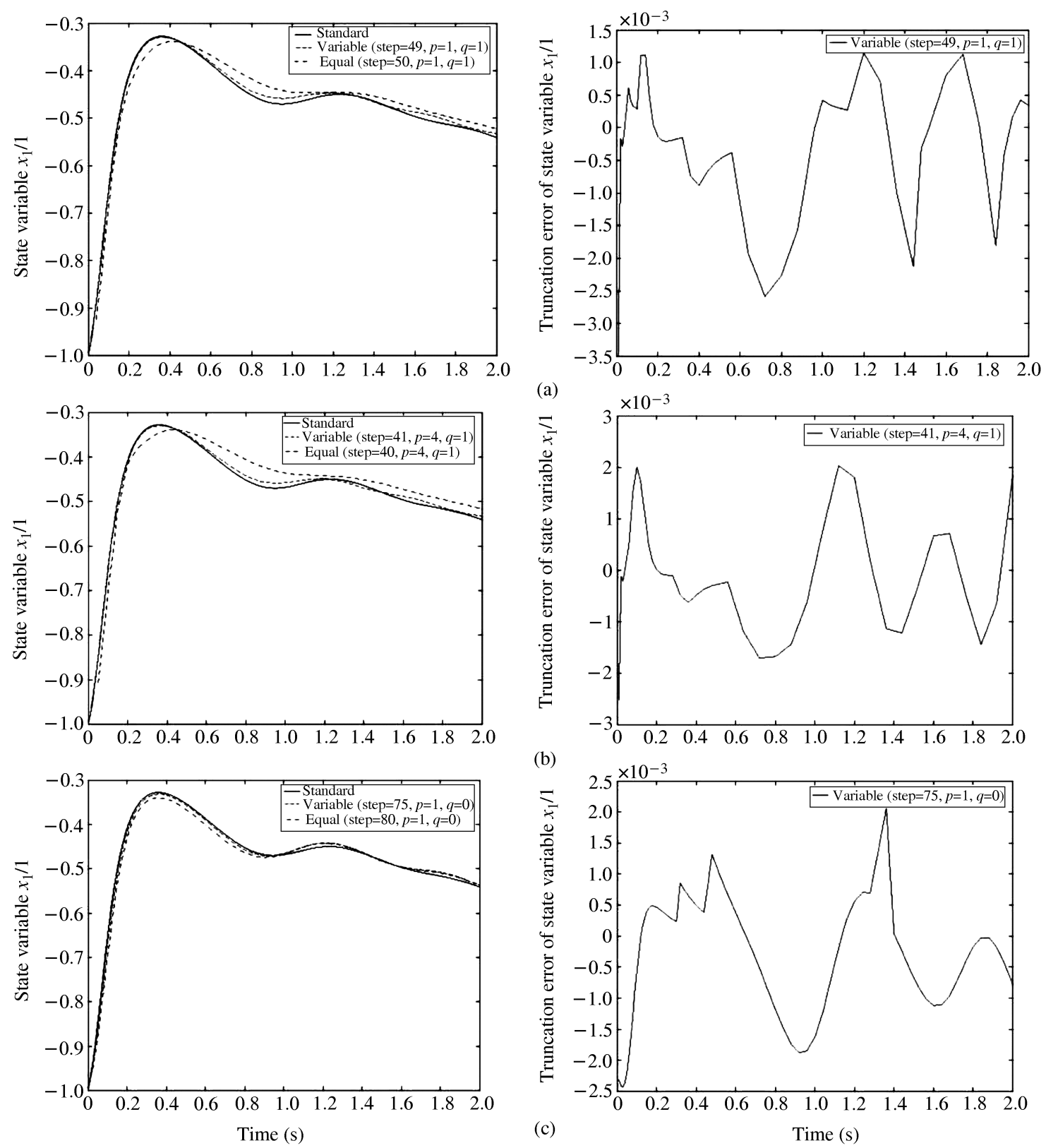

Figure 3 Variable $x_{1}$ and truncation $e_{x 1}$ over time. (a) Situation $1(p=1, q=1)$; (b) situation $2(p=4, q=1)$; (c) situation $3(p=1, q=0)$.

error, and the changes of truncation error $e_{x 1}$ on the simulation time period are shown in Figures 3(a), (b) and (c). As shown in the figures, the variable-step simulations based on the proposed method for truncation error calculation have better performance in both efficiency and accuracy than those by using constant step-size simulation of usual combinative algorithm [17].

\section{Conclusions}

Collaborative simulation is a popular and effective approach for modeling and simulation of complex systems. It aims to achieve the multidisciplinary simulation and optimization of complex engineering systems, by taking into account the coupling modeling and collaborative computation between multidisciplinary physical systems. For the simulation of a single system without interaction with others, the variable-step algorithm is designed to restrict the step size based on error estimation, and it is becoming 
increasingly mature in terms of both theoretical investigations and practical applications. However, no mature methods are available up to now to solve the issues of variable-step computation and error estimation in multidisciplinary collaborative simulation. This work is motivated to develop variable-step algorithms for the collaborative simulation of multidisciplinary coupling systems.

Based on the analysis of variable-step algorithms for single-system simulation, a variable-step algorithm is proposed based on Richardson extrapolation for the collaborative simulation of multidisciplinary coupling systems. The calculation method for local truncation error of the collaborative simulation system is also developed, and formulas for estimating the local truncation error are derived through theoretical analysis in the different cases by using integration methods and interpolation technologies, respectively. This research provides a new way for solving calculation and estimation problems of the local truncation errors of multidisciplinary coupling systems, which lays the foundation of variable-step collaborative simulation.

\section{Acknowledgements}

This work was supported by the National Natural Science Foundation of China (Grant Nos. 61074110, 60874071, 60834004). The authors would like to thank Dr. Xiaomeng Chang for her contribution to this paper.

\section{References}

1 Shephard M S, Beall M W, O'Bara R M, et al. Toward simulation-based design. Finite Elem Anal Des, 2004, 40: $1575-1598$

2 Steven D P, Oleg W. An efficient multirate simulation technique for power-electronic-based systems. IEEE Trans Power Syst, 2004, 19: 399-409

3 Li G, Song S, Wu C. Generalized gradient projection neural networks for nonsmooth optimization problems. Sci China Inf Sci, 2010, 53: 990-1005

4 Zhang H. A solution of multidisciplinary collaborative simulation for complex engineering systems in a distributed heterogeneous environment. Sci China Ser F-Inf Sci, 2009, 52: 1848-1862

5 Zhang H, Wang H, Chen D, et al. A model-driven approach to multidisciplinary collaborative simulation for virtual product development. Adv Eng Inf, 2010, 24: 167-179

6 Senin N, Wallace D, Borland N. Distributed object-based modeling in design simulation marketplace. Trans ASME J Mech Des, 2003, 125: 2-13

7 Wang H, Zhang H. A distributed and interactive system to integrated design and simulation for collaborative product development. Robot Comput-Integr Manuf, 2010, 26: 778-789

8 Wang H, Johnson A, Zhang H, et al. Towards a collaborative modeling and simulation platform on the Internet. Adv Eng Inf, 2010, 24: 208-218

9 Wang H, Zhang H. An integrated and collaborative approach for complex product development in distributed heterogeneous environment. Int J Prod Res, 2008, 46: 2345-2361

10 Felippa C A, Park K C, Farhat C. Partitioned analysis of coupled mechanical systems. Comput Meth Appl Mech Eng, 2001, 190: 3247-3270

11 Shome S S, Haug E J, Jay L O. Dual-rate integration using partitioned Runge-Kutta methods for mechanical systems with interacting subsystems. Mech Based Des Struct Mach, 2004, 32: 253-282

12 Arnold M. Multi-rate time integration for large scale multibody system models. In: IUTAM Symposium on Multiscale Problems in Multibody System Contacts. Dordrecht: Springer, 2007: 1-10

13 Arnold M. Numerical methods for simulation in applied dynamics. In: Simulation Techniques for Applied Dynamics. New York: Springer, 2009. 191-246

14 Gear C W, Well D R. Multirate linear multistep methods. BIT, 1984, 24: 484-502

15 Liu D, Fei J. Digital Simulation Algorithms for Dynamic Systems (in Chinese). Beijing: Science Press, 2000

16 Fei J. Convergence and convergence order of decomposition methods with interpolations for numerical initial value problems of ordinary differential equations (in Chinese). Numer Calculat Comput Appl, 1994, 12: 209-218

17 Liang S, Zhang H. A novel combinative algorithm for multidisciplinary collaborative simulation. In: Proceedings of the 12th International Conference on CSCWD. Xi'an, China, 2008. 104-109

18 Xiong G, Song A. Algorithm selection and step size control of digital simulation (in Chinese). Comput Simul, 1985, 4: $1-5$

19 Schiehlen W, Scholz C. Step size control of simulator coupling for multibody systems. In: 2nd Workshop on CoSimulation. 2001

20 Kübler R, Schiehlen W. Two methods of simulator coupling. Math Comput Model Dyn Syst, 2000, 6: 93-113 